\title{
A search for rapid oscillations in chemically peculiar A-type stars
}

\author{
G. Handler and E. Paunzen \\ Institut für Astronomie, Universität Wien, Türkenschanzstraße 17, A-1180 Wien, Austria
}

Received August 27; accepted September 25, 1998

\begin{abstract}
In 1995 we initiated a Northern Hemisphere survey for rapidly oscillating Ap stars. This paper presents the results including one new roAp star (HD 122970), the confirmation of rapid oscillations of HD 99563 and apparent null results for other stars.

Using Hipparcos data a statistical analysis of the absolute magnitudes and galactic distributions of all known roAp and noAp stars (also taken from the literature) was made.

A systematic trend for most of the program stars in a $M_{V}$ vs. $\beta$ (index of the Strömgren uvby $\beta$ system) diagram was detected leading to the conclusion that $\beta$ is systematically influenced by the chemical peculiarity and/or magnetic field.

Three roAp stars are outside the $\delta$ Scuti instability strip which implies that the driving mechanism of the two classes of pulsating star is different. This is also suggested by new pulsation models. No statistical difference between the galactic distribution of roAp and noAp stars was found.
\end{abstract}

Key words: stars: chemically peculiar — stars: oscillations

\section{Introduction}

Among the magnetic chemically peculiar A-type stars, the rapidly oscillating Ap (roAp) stars are quite outstanding. This group exhibits rapid photometric and spectroscopic variations with periods of minutes and very small amplitudes $\left(A_{B} \leq 10 \mathrm{mmag}\right)$. These oscillations are probably low spherical degree, high overtone p-modes. Using the (in general) rich oscillation spectra, several important astrophysical parameters such as the (asteroseismological) luminosity, the rotational period, the magnetic field strength and the atmospheric structure can be inferred.

Since the early eighties, the South African working group has spent a lot of time in detecting new roAp stars.

Send offprint requests to: G. Handler
They discovered more than twenty new roAps (e.g. see Martinez \& Kurtz 1995) and published also an extensive list of null results (Martinez \& Kurtz 1994). Several surveys in the Northern Hemisphere were also devoted to find new members of this group (e.g. Heller \& Kramer 1988; Nelson \& Kreidl 1993). Unfortunately, only one new roAp star (HD 176232, 10 Aql) was detected. Recently, Dorokhova \& Dorokhov (1998) announced the discovery of a second roAp star (HD 99563) discovered at a Northern Hemisphere observatory.

This situation leads to a dilemma when trying to make a statistically sound analysis for this group. Two main problems arise:

- A bias is naturally introduced having only (beside two) southern roAp stars.

- Follow-up observations (e.g. high resolution spectroscopy) need to be performed in the Southern Hemisphere where telescope time is rare.

Since only one observatory (Mt. Dushak-Erekdag, Central Asia; Dorokhova \& Dorokhov 1998) regularly searches for new roAp stars, we decided to initiate a Northern Hemisphere survey for roAp stars in 1995. Observations are carried out with one of the twin $0.75 \mathrm{~m}$ Austrian Automatic Photoelectric Telescopes at Fairborn Observatory and at McDonald Observatory.

In this paper we report the discovery of one new equatorial roAp star (HD 122970), the confirmation that HD 99563 shows rapid oscillations and the apparent null results of our survey. With the help of Hipparcos data we examine the absolute magnitudes and galactic distribution of all investigated stars.

\section{Target selection and observations}

Since the main aim of our program was a statistical exploration of the roAp phenomenon, we attempted to bias our target selection towards the detection of new roAp stars. Most of these objects are cool $\mathrm{SrCrEu}$ stars. Regrettably, systematic spectral classifications for 
Northern Hemisphere objects are still missing in the literature. Therefore we chose a different approach.

Nelson \& Kreidl (1993) explored the positions of pulsating and nonpulsating Ap stars in several color-color diagrams for the Strömgren and Geneva photometric systems. While they did not find a clear separation of the two groups, the Strömgren $\left[m_{1}\right],\left[c_{1}\right]$ and Geneva $\Delta,[g]$ diagrams appear useful to select promising roAp candidates. Consequently, we searched existing Strömgren (Hauck \& Mermilliod 1998) and Geneva catalogues (Rufener 1989) for potential target objects.

Since this procedure does not allow one to distinguish between Am and Ap stars, we attempted to find spectral classifications for the objects of interest in the literature. Consequently, priorities were assigned to the different stars and observations were performed according to them.

Three telescopes at two observatories were employed for our survey: the $0.9 \mathrm{~m}$ and $2.1 \mathrm{~m}$ telescopes at McDonald Observatory, Ft. Davis, Texas together with a standard two-channel photometer (Grauer \& Bond 1981) and one of the twin $0.75 \mathrm{~m}$ Austrian Automatic Photoelectric Telescopes (APT, Strassmeier et al. 1997) at Fairborn Observatory, Washington Camp, Arizona with a single-channel photometer.

The observations (see Table 1 for an overview) were carried out as continuous 10-second integrations through a Johnson $B$ filter. Large apertures $\left(>35^{\prime \prime}\right)$ were employed to minimize the contributions of seeing and guiding ${ }^{1}$.

The reductions were performed using the standard technique for roAp star observations (e.g. Martinez 1993), including some low-frequency filtering in the presence of sky transparency variations by low-order polynomial fits or involving the Channel 2 comparison star, if found appropriate. The final reduced light curves were searched for rapid oscillations by means of a standard Fourier technique (Breger 1990). Amplitude spectra of our runs are displayed in Figs. 1-3 (see below for more details).

\subsection{Comments on individual stars}

BD+39 654A: This star was our most promising roAp candidate. It has been classified at $\mathrm{SrCrEu}$ by Bidelman (1983). Schneider's (1986) Strömgren photometry yields $(b-y)_{0}=0.136, \delta m_{1}=-0.095$ and $\delta c_{1}=-0.267$, while Geneva photometry (Burki et al. 1998) results in $[\Delta]=0.163$ and $[g]=0.162$. All these features are typical for roAp stars. However, BD+39 654A did not show any rapid variability, but ironically, its Channel 2 comparison star, HD 17892, turned out to be a new $\delta$ Scuti variable

\footnotetext{
${ }^{1}$ For HD $83965 \mathrm{~B}$, which has a close bright companion, a $18^{\prime \prime}$ aperture was used to exclude the companion; continuous guiding on the Channel 2 star was performed to check telescope tracking.
}
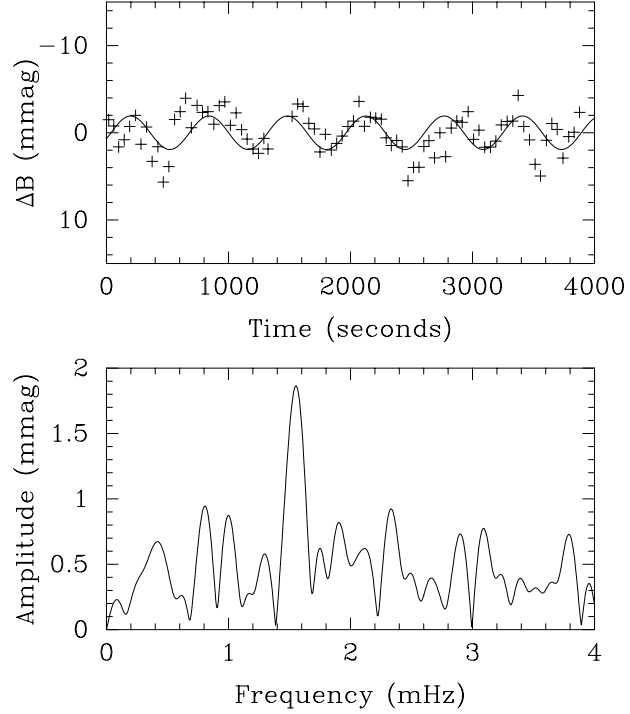

Fig. 1. Upper panel: a portion of our light curve of HD 99563. Lower panel: The amplitude spectrum of the whole run
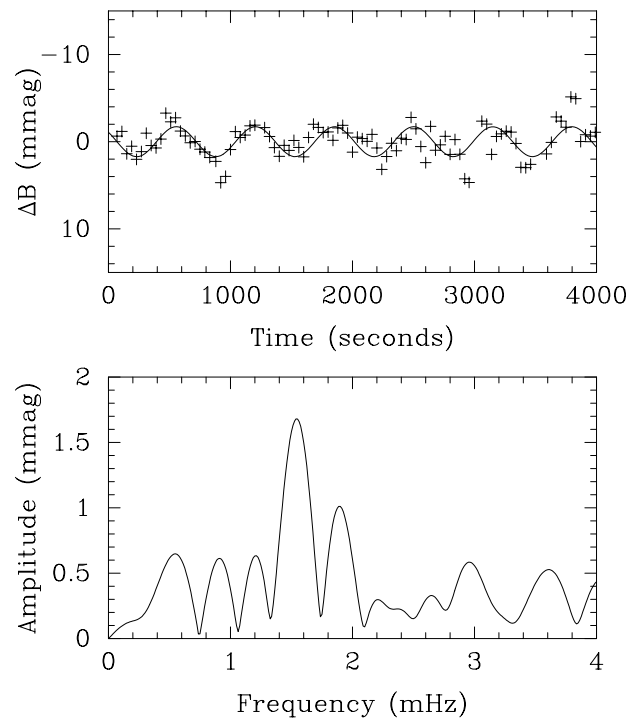

Fig. 2. Upper panel: the discovery light curve of HD 122970. Lower panel: The amplitude spectrum of this run

(Handler 1998). In any case, we consider it worthwhile to continue testing BD+39 654A for rapid oscillations.

BD+40 175A: This is the only object for which we have no color photometry available. However, its spectral classification ( $\mathrm{SrCrEu}$, Bidelman 1985) and its effective temperature estimate $(7100 \pm 400 \mathrm{~K}$, Babel \& North 1997) make it a good roAp candidate.

We took a short run under non-ideal photometric conditions, where this target seemed to show rapid oscillations with an amplitude of 6 mmag and a period of 9.1 minutes. Two further runs, one with the McDonald $2.1 \mathrm{~m}$ telescope considerably decreasing scintillation noise, did not confirm this possible variability. We suggest further observations of $\mathrm{BD}+40175 \mathrm{~A}$ as well. 
Table 1. Journal of the observations

\begin{tabular}{lrrlccc}
\hline $\begin{array}{l}\text { Date } \\
(\mathrm{UT})\end{array}$ & \multicolumn{1}{c}{$\begin{array}{c}\text { Start } \\
(\mathrm{UT})\end{array}$} & $\begin{array}{c}\text { Length } \\
(\text { hrs })\end{array}$ & Target & $V$ & Observatory & Comment \\
\hline 19 Jan. 1998 & $1: 32: 00$ & 0.68 & BD+40 175A & 8.9 & McD & roAp? \\
23 Jan. 1998 & $1: 52: 30$ & 0.58 & BD+40 175A & & McD & \\
26 Jan. 1998 & $1: 43: 20$ & 1.04 & BD+40 175A & & McD $(2.1 \mathrm{~m})$ & \\
17 Jan. 1998 & $1: 27: 00$ & 3.08 & BD+39 654A & 9.48 & McD & \\
03 Feb. 1996 & $1: 32: 30$ & 1.79 & HD 31437 & 6.88 & McD & \\
06 Feb. 1996 & $1: 39: 00$ & 3.16 & HD 33505 & 9.21 & McD & \\
05 Feb. 1996 & $1: 30: 10$ & 2.94 & HD 35353 & 7.68 & McD & \\
28 Mar. 1998 & $2: 07: 29$ & 2.99 & HD 51596 & 7.52 & Fairborn & \\
10 Feb. 1996 & $1: 40: 20$ & 1.28 & HD 57955 & 7.68 & McD & \\
22 Jan. 1998 & $11: 19: 40$ & 1.03 & HD 83965B & 11.87 & McD & roAp \\
22 Mar. 1998 & $4: 02: 45$ & 2.07 & HD 99563 & 8.32 & Fairborn & \\
23 Feb. 1998 & $10: 46: 50$ & 1.39 & HD 108449 & 8.31 & McD & \\
29 Jan. 1998 & $11: 08: 00$ & 1.07 & HD 108873 & 9.56 & McD (2.1 m) & \\
25 Feb. 1998 & $10: 36: 00$ & 1.53 & HD 112515A & 8.51 & McD & \\
28 Mar. 1998 & $5: 12: 23$ & 1.81 & HD 113894 & 8.55 & Fairborn & \\
15 Jan. 1998 & $11: 48: 20$ & 1.00 & HD 122970 & 8.31 & McD & roAp \\
17 Jan. 1998 & $11: 40: 20$ & 1.24 & HD 122970 & & McD & \\
18 Jan. 1998 & $11: 03: 40$ & 1.00 & HD 122970 & & McD & \\
19 Jan. 1998 & $11: 32: 10$ & 1.34 & HD 139478 & 6.74 & McD & \\
09 Aug. 1995 & $2: 42: 50$ & 0.81 & HD 151839 & 9.28 & McD & \\
12 Aug. 1995 & $10: 28: 55$ & 0.59 & HD 223839 & 7.28 & McD & \\
\hline
\end{tabular}

HD 99563: Dorokhova \& Dorokhov (1998) announced the variability of this object with a period of about $11.2 \mathrm{~min}-$ utes. We reobserved this star with the APT. The amplitude spectrum of our data shows a dominant peak with an amplitude of 2.0 mmag with a period of 10.7 minutes (Fig. 1). Therefore we consider the roAp nature of this star to be confirmed.

HD 122970: This is a new roAp star. We discovered rapid oscillations with an amplitude of $2.0 \mathrm{mmag}$ and a period of 11.1 minutes in the first night we observed it (Fig. 2). This behaviour was confirmed during two subsequent runs. This star was followed up with multisite photometric and single-site spectroscopic observations; the results of this effort will be reported in a subsequent paper.

\section{Analysis}

For our explorations of possible group properties of roAp stars, we only considered stars which have been tested for rapid light variations. The list of known roAp stars was taken from Kurtz \& Martinez (1993), five additional roAp stars were added, namely: HD 9289, HD 99563, HD 122970, HD 185256, HD 213637 resulting in 31 roAp stars known at the writing of this paper. The null results (noAp) were taken from Martinez \& Kurtz (1994), including results from other surveys (see Tables 1 and 2 in Martinez \& Kurtz 1994) as well. No further references on null results were found in the literature. We have to emphasize that the temperature range for the noAp stars $(15000-6000 \mathrm{~K})$ is wider than that of the roAp stars
$(8500-6500 \mathrm{~K})$, i.e. some objects desgined as noAp may in fact be Bp stars.

We have used the Hipparcos and Tycho catalogues (Perryman et al. 1997) to retrieve parallaxes and apparent places for all stars. For the given sample (31 roAp and 229 noAp stars) only stars with $\frac{\sigma(\pi)}{\pi}<0.18\left(\sigma\left(M_{V}\right)<\right.$ $0.3 \mathrm{mag})$ were considered. A Lutz-Kelker correction according to Koen (1992) was applied. Because of the chosen error limit for the parallax, the Lutz-Kelker correction is very small for all program stars. We have not applied any correction for interstellar extinction because of two reasons:

- Most of our program stars are within 100 pc resulting in negligible reddening.

- Values for $(B-V)_{0},(b-y)_{0}$ and $(B 2-V 1)_{0}$ are derived for "normal" type stars only. It has been proven that these calibrations are, in general, not valid for magnetic chemically peculiar stars ("blueing effect").

Taking all considerations into account we are left with 12 roAp and 54 noAp stars. Figure 4 shows the $M_{V}($ Hip $)$ vs. $\beta$ diagram. There are several points evident from the figure:

- All roAp stars behave "normal", in the sense that the fall in the region of other main sequence stars.

- Most of the noAp stars are above the normality line. This could mean that either the $\beta$-values are systematically too large or that the absolute magnitude is systematically too bright. Latter cannot be explained by reddening since it would even more brighten the stars. 

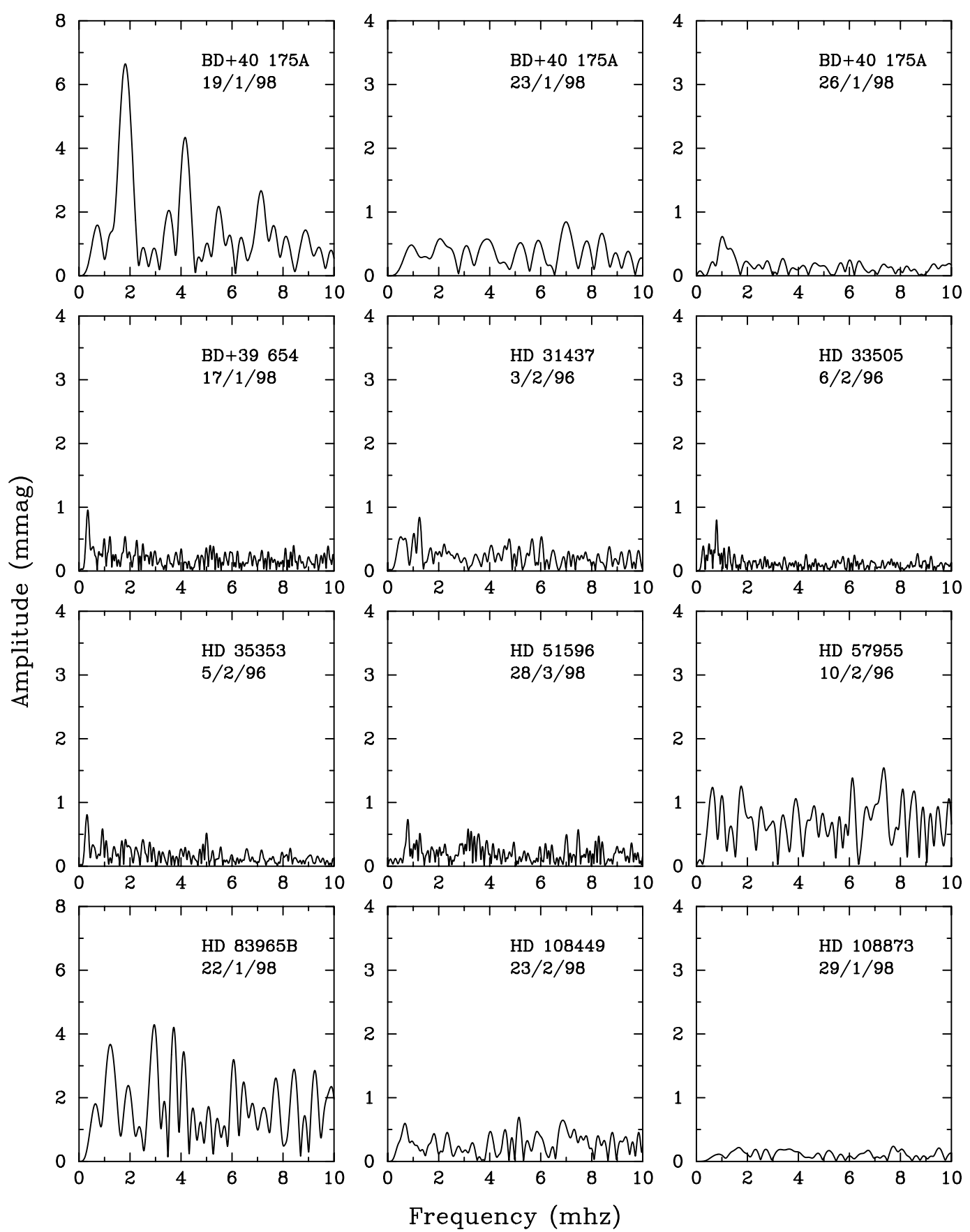

Fig. 3. Amplitude spectra of our null results. Note the larger ordinate scales for the first run on BD+40 $175 \mathrm{~A}$ and for the run on HD $83965 \mathrm{~B}$

It is worthwhile to note that the roAp stars are separated from the noAp's in Fig. 4: they are all rather cool mainsequence objects. Only a few stars, for which no rapid oscillations have been detected, are located close to the pulsators (HD 15233, HD 25354, HD 35353, HD 62140, HD 115708, HD 154708, HD 170397 and HD 188854). HD 25354 and HD 170397 are probably Bp stars (as judged from their Stromgren indices), while HD 35353 is poorly observed and there is no spectral classification available (hence it could be an Am star). The remaining three stars are well observed and from Fig. 4 there is no reason why they should not be roAp stars, except that HD 15233 and HD 188854 may be too evolved. We note 


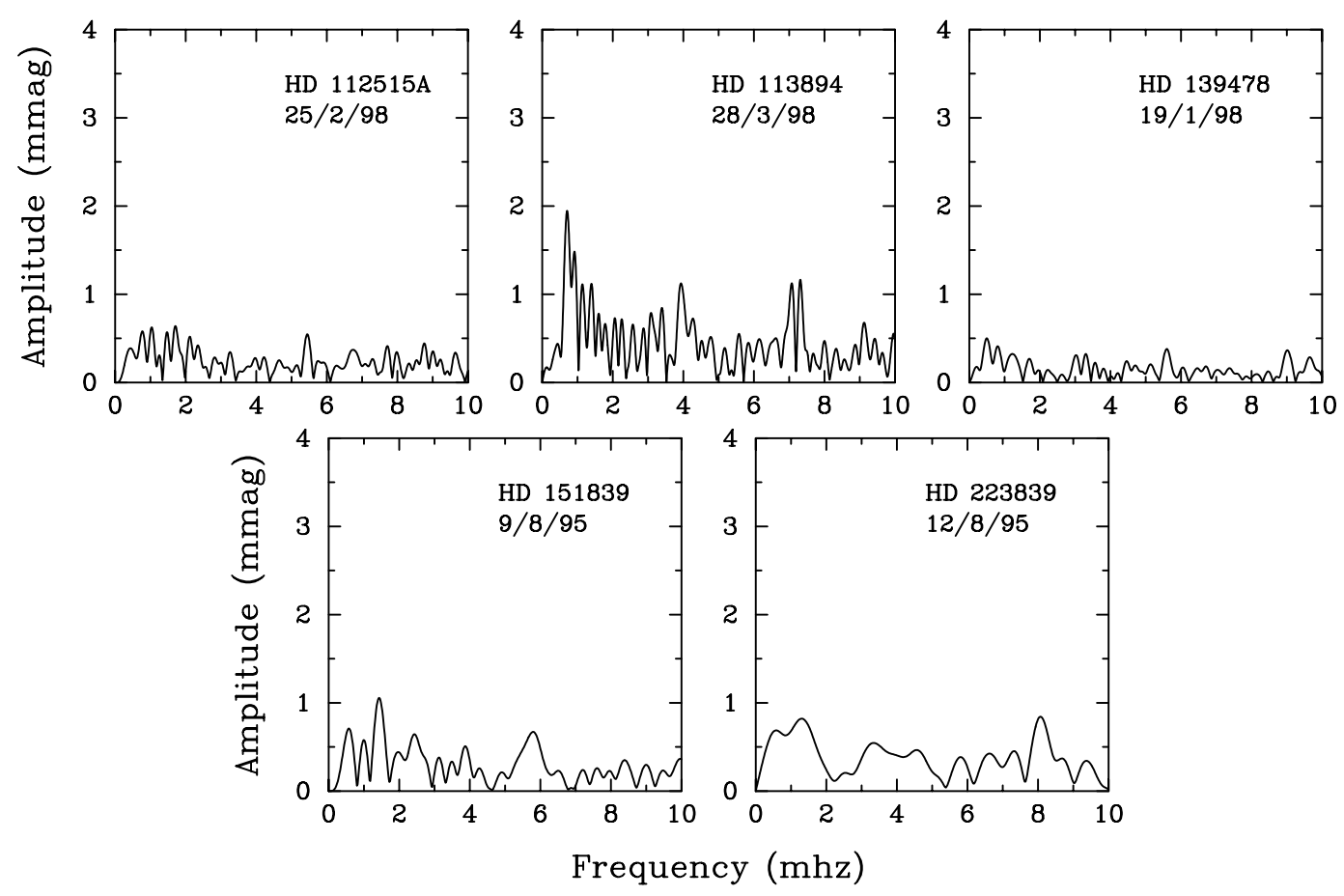

Fig. 3. continued

that the most famous noAp star, HD 137909 ( $\beta$ CrB), appears to be hotter and more evolved than all roAp stars.

There is more evidence that effective temperatures for Ap stars determined from $\beta$ are systematically too high. Alonso et al. (1996) compared $T_{\text {eff }}$ values from the Infrared Flux method with $\beta$ measurements for a large range of metallicities. They found a clear trend of increasing $\beta$ with increasing metallicity for fixed $T_{\text {eff }}$.

Matthews et al. (1998) compared asteroseismological parallaxes with those measured by Hipparcos. They noted that the Hipparcos parallaxes are generally larger than the asteroseismic ones ${ }^{2}$, and suggested this may be due to systematically incorrect effective temperatures estimated from $\beta$.

Some more support for this idea comes from a comparison of $\beta$-temperatures and those determined by model atmosphere analysis for a number of roAp stars (Gelbmann 1998 and references therein). This is summarized in Fig. 5. We find that the best fitting model atmospheres generally point towards lower effective temperatures. The

\footnotetext{
2 There is a caveat: the seismological parallaxes are inferred by applying asymptotic theory. Although roAp stars pulsate in high radial overtones, the frequency spacing for these consecutive overtones is still smaller than the asymptotic one, resulting in (by several percent) systematically smaller seismological parallaxes. Furthermore, model calculations by Gautschy et al. (1998) show that significant deviations of observed frequency spacings from the asymptotic values can occur due to nonadiabatic effects.
}

mean temperature difference is $-80 \pm 90 \mathrm{~K}$, which is not significant. However, this analysis can be improved when higher resolution spectra of (ro)Ap stars become available (allowing to determine more accurate temperatures) and a larger sample is investigated.

The Hipparcos parallaxes strongly suggest that the roAp stars are main-sequence objects. This is supported by the study of Gómez et al. (1998), who examined the positions of Bp-Ap stars in the HR diagram. They show that these stars are on the main sequence, and this also holds for the $\mathrm{SrCrEu}$ stars, i.e. the spectral subgroup containing the roAp stars. When examining the effective temperatures of roAp stars one obtains from $\beta$ by using the calibrations of Moon \& Dworetsky (1985), it can be noted that three ${ }^{3}$ out of the 31 roAp stars (HD 122970 , HD 213637, HD 217522) are clearly outside the cool border of the $\delta$ Scuti instability strip, especially since these temperatures are presumably overestimates.

For some years, it has been believed that the $\delta$ Scuti and roAp instability strips coincide, which has been taken as an argument that the driving mechanism for these two classes of pulsating star could be the same (partial $\mathrm{He}^{+}$ionisation). Very recently, Gautschy et al. (1998) presented model calculations, which led them to suggest that the actual driving of roAp pulsations might be due to

\footnotetext{
${ }^{3}$ We deliberately do not consider HD 101065 here, since this object is so peculiar that its effective temperature cannot even be estimated with some confidence.
} 


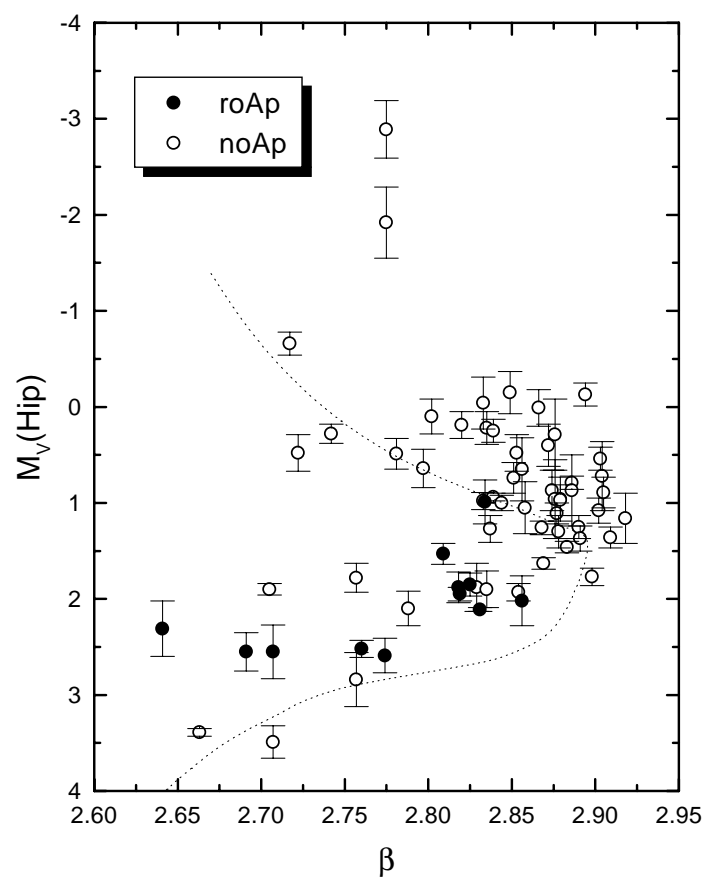

Fig. 4. $M_{V}($ Hip $)$ vs. $\beta$ diagram for 12 roAp (filled cricles) and 54 noAp (open circles) stars, the normality line is from Crawford (1979)

partial $\mathrm{H} / \mathrm{He}$ ionisation. They obtained overstable highorder modes by assuming that these stars have chromospheres and therefore a temperature inversion in their atmospheres. Under these assumptions their models showed roAp pulsations outside the cool edge of the $\delta$ Scuti instability strip, and hence they can explain why the three stars mentioned above do pulsate.

In Fig. 6 we present a $l$ vs. $b$ diagram for all program stars. Beside the "southern hemisphere effect" no systematic differences of the galactic distribution for roAp and noAp stars are evident.

\section{Summary}

We have presented first results of our Northern Hemisphere survey for roAp stars including photometric observations for 17 stars. One star (HD 122970) turned out to be a new roAp star, furthermore rapid oscillations for HD 99563 were confirmed.

Including all program stars from other surveys, a statistical analysis of absolute magnitudes (using Hipparcos data) and galactic coordinates has been made. The galactic distribution of roAp and noAp stars show no significant differences whereas the absolute magnitudes and/or the

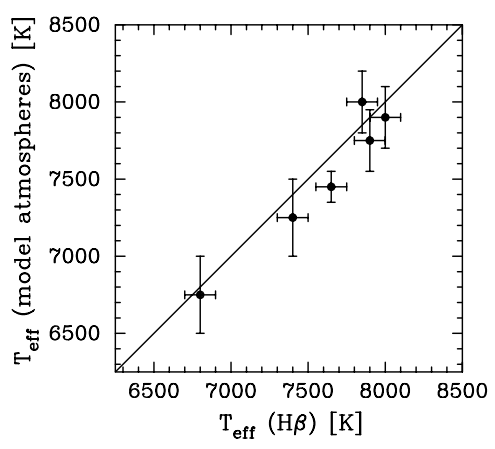

Fig. 5. Effective temperatures for six roAp stars from $\mathrm{H} \beta$ photometry compared with those from model atmosphere analysis. The solid line corresponds to exact agreement. Except for one star (HD 166473), the $\mathrm{H} \beta$ temperatures are always higher

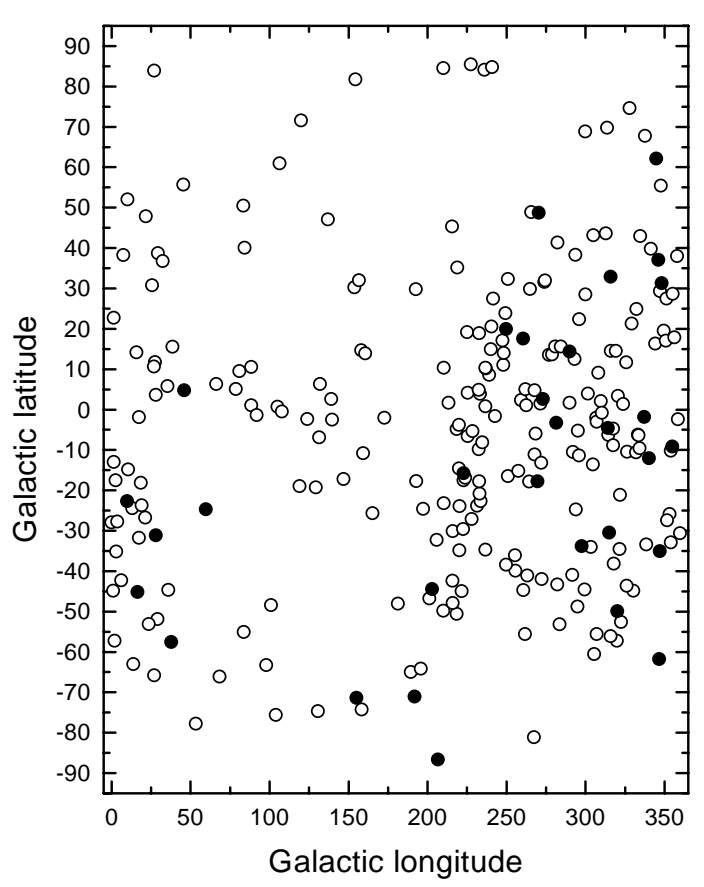

Fig. 6. $l$ vs. $b$ diagram for all program stars

effective temperatures show systematic trends. Evidence is given that the effective temperatures for roAp stars are systematically too high when "standard" calibrations for $\mathrm{H} \beta$ photometry are applied.

Absolute magnitudes from Hipparcos data for roAp stars establish them as main sequence objects. Three objects are outside the $\delta$ Scuti instability strip which points towards a different excitation mechanism of the two classes 
of pulsating star. This is also suggested by new pulsation models.

Acknowledgements. The Austrian Fonds zur Förderung der wissenschaftlichen Forschung partially supported this work under grants S7303-AST and S7304-AST. APT observations were supported under grant $S 7301-A S T$ to K. G. Strassmeier.

\section{References}

Alonso A., Arribas S., Martinez-Roger C., 1996, A\&A 313, 873

Babel J., North P., 1997, A\&A 325, 195

Bidelman W.P., 1983, AJ 88, 1182

Bidelman W.P., 1985, AJ 90, 341

Breger M., 1990, Communications in Asteroseismology 20, 1 (University of Vienna)

Burki G., et al., 1998 (in preparation)

Crawford D.L., 1979, AJ 84, 1858

Dorokhova T.N., Dorokhov N.I., 1998, in Proceedings of the 26th meeting of the European Working Group on CP stars, North P., Schnell A., Žižňovský J. (eds.), Contributions of the Astronomical Observatory Skalnaté Pleso. Slovak Academy of Sciences, p. 338

Gautschy A., Saio H., Harzenmoser H., 1998, MNRAS (in press)
Gelbmann M.J., 1998, PhD thesis, University of Vienna Gómez A.E., Luri X., Grenier S., et al., 1998, A\&A 336, 953 Grauer A.D., Bond H.E., 1981, PASP 93, 388

Handler G., 1998, IBVS 4550

Hauck B., Mermilliod M., 1998, A\&AS 129, 431

Heller C.H., Kramer K.S., 1988, PASP 100, 583

Koen C., 1992, MNRAS 256, 65

Kurtz D.W., Martinez P., 1993, IBVS 3966

Martinez P., 1993, in Precision Photometry, Kilkenny D. et al. (eds.). South African Astronomical Observatory, p. 134

Martinez P., Kurtz D.W., 1994, MNRAS 271, 129

Martinez P., Kurtz D.W., 1995, Ap\&SS 230, 29

Matthews J.M., Kurtz D.W., Martinez P., 1998, ApJ (in press)

Moon T.T., Dworetsky M.M., 1985, MNRAS 217, 305

Nelson M.J., Kreidl T.J., 1993, AJ 105, 1903

Perryman M.A.C., Høg E., Kovalevsky J., Lindegren L., Turon C., 1997, The Hipparcos and Tycho Catalogues, ESA SP1200

Rufener F., 1989, A\&AS 78, 469

Schneider H., 1986, A\&A 161, 203

Strassmeier K.G., Boyd L.J., Epand D.H., Granzer T., 1997, PASP 109, 697 